\title{
Evaluation of the coverage and depth of transcriptome by RNA-Seq in chickens
}

\author{
Ying Wang ${ }^{1}$, Noushin Ghaffari ${ }^{2,3}$, Charles D Johnson², Ulisses M Braga-Neto ${ }^{3}$, Hui Wang ${ }^{4}$, Rui Chen ${ }^{4}$, \\ Huaijun Zhou $^{1 *}$ \\ From Eighth Annual MCBIOS Conference. Computational Biology and Bioinformatics for a New Decade \\ College Station, TX, USA. 1-2 April 2011
}

\begin{abstract}
Background: RNA-Seq is the recently developed high-throughput sequencing technology for profiling the entire transcriptome in any organism. It has several major advantages over current hybridization-based approach such as microarrays. However, the cost per sample by RNA-Seq is still prohibitive for most laboratories. With continued improvement in sequence output, it would be cost-effective if multiple samples are multiplexed and sequenced in a single lane with sufficient transcriptome coverage. The objective of this analysis is to evaluate what sequencing depth might be sufficient to interrogate gene expression profiling in the chicken by RNA-Seq.

Results: Two cDNA libraries from chicken lungs were sequenced initially, and 4.9 million (M) and $1.6 \mathrm{M}$ (60 bp) reads were generated, respectively. With significant improvements in sequencing technology, two technical replicate CDNA libraries were re-sequenced. Totals of $29.6 \mathrm{M}$ and $28.7 \mathrm{M}(75 \mathrm{bp}$ ) reads were obtained with the two samples. More than $90 \%$ of annotated genes were detected in the data sets with 28.7-29.6 M reads, while only $68 \%$ of genes were detected in the data set with $1.6 \mathrm{M}$ reads. The correlation coefficients of gene expression between technical replicates within the same sample were 0.9458 and 0.8442 . To evaluate the appropriate depth needed for mRNA profiling, a random sampling method was used to generate different number of reads from each sample. There was a significant increase in correlation coefficients from a sequencing depth of $1.6 \mathrm{M}$ to $10 \mathrm{M}$ for all genes except highly abundant genes. No significant improvement was observed from the depth of $10 \mathrm{M}$ to $20 \mathrm{M}(75 \mathrm{bp})$ reads.

Conclusion: The analysis from the current study demonstrated that $30 \mathrm{M}(75 \mathrm{bp})$ reads is sufficient to detect all annotated genes in chicken lungs. Ten million (75 bp) reads could detect about $80 \%$ of annotated chicken genes, and RNA-Seq at this depth can serve as a replacement of microarray technology. Furthermore, the depth of sequencing had a significant impact on measuring gene expression of low abundant genes. Finally, the combination of experimental and simulation approaches is a powerful approach to address the relationship between the depth of sequencing and transcriptome coverage.
\end{abstract}

\section{Background}

The transcriptome catalogues the complete set of transcripts in a cell. Transcriptomic regulation is critical to all physiological, developmental and pathological processes [1], and mRNA expression profiles can represent the characteristics of a cell at a specific state and help to

\footnotetext{
* Correspondence: hjzhou@poultry.tamu.edu

'Department of Poultry Science, Texas A \&M University College Station, TX 77843-2472, USA

Full list of author information is available at the end of the article
}

govern its present and future activities [2]. The profiles of a transcriptome in terms of alterations in response to specific biological stimuli provides valuable insights for interpreting functional elements of the genome, revealing the molecular constituents of cells, and also understanding developmental and disease processes.

Different types of technologies have been developed to interrogate transcript abundance, including hybridization-based and sequencing-based approaches. Hybridization-based microarrays have been the primary
C Biomed Central

() 2011 Wang et al; licensee BioMed Central Ltd. This is an open access article distributed under the terms of the Creative Commons Attribution License (http://creativecommons.org/licenses/by/2.0), which permits unrestricted use, distribution, and reproduction in any medium, provided the original work is properly cited. 
transcriptomic high-throughput tool for almost two decades, which has accelerated the study of transcriptome analysis by profiling thousands of genes simultaneously [3]. However, microarray technology has several limitations including: indirect quantification by hybridization-signal intensities [4], background and cross-hybridization problems [5] and reproducibility issues [6]. The development of next generation sequencing with improved qualitative and quantitative measurements holds great promise in transcriptome analysis.

RNA-Seq is a recently developed approach to map and quantify transcriptomes by digitally recording how frequently each transcript is represented in a sequence sample. After poly (A) selection, RNA is fragmented to small fragments and converted into a cDNA library, which provides a simple and more comprehensive way to measure transcriptome composition and to discover new genes by high-throughput sequencing without bacterial cloning of cDNA input [2]. Studies using this technology have already altered our views regarding the extent and complexity of transcriptomes in an organism and dramatically improved our understanding of transcriptome. RNA-Seq has several advantages over micorarrays including: 1) RNA-Seq is not dependent on prior knowledge about the target sequence; 2) It has a large dynamic range and sensitivity due to its digital nature, which is especially important for highly abundant and extremely low abundant genes; 3) The survey of a transcriptome is more accurate because the quantification of each transcript is directly based on digital counts of the transcript. Therefore, RNA-Seq offers both single-base resolution for annotation and "digital" quantification at the RNA level, which allows the entire transcriptome to be analyzed in a highthroughput and quantitative manner [7]. However, the expense per sample for RNA- Seq is still a limiting factor in preventing researchers from sequencing multiple biological replicates per group, which are needed for statistically-significant analysis. It is common to adopt a pooling strategy to reduce the cost for RNA-Seq studies [8]. With the continued enhancement of sequencing output and the development of multiplex labelling techniques, the cost per sample could be significantly reduced if several samples are multiplexed and sequenced in the same lane, given sufficient transcriptome coverage per sample. Therefore, it is imperative to address the trade-off between the depth of RNASeq and the coverage of the transcriptome in an organism. The objective of this study was to evaluate what coverage or sequencing depth of transcriptome would be sufficient to interrogate gene expression profiling in the chicken by RNA-Seq.

\section{Methods}

\section{RNA preparation}

Total RNA was isolated from four chicken lungs from two genetic chicken lines (two samples per line) by Trizol (Invitrogen, Carlsbad, CA) according to the manufacturer's protocol. Two RNA samples from the same line were pooled to generate totals of two pooled RNA samples (Sample1 and Sample2). DNase I (Ambion, Austin, TX) digestion was carried out after RNA isolation and the RNA concentration and purity were determined by measuring absorbance at $260 \mathrm{~nm}$ and A260/ A280 ratio using a NanoDrop ND-1000 spectrophotometer (Nano-drop Technologies, Wilmington, DE). RNA samples were stored at $-80^{\circ} \mathrm{C}$ until further use.

\section{cDNA library preparation and sequencing by RNA-Seq}

Total RNA $(7 \mu \mathrm{g})$ was subjected to two rounds of hybridization to oligo (dT) beads (Invitrogen, Carlsbad, CA) to enrich mRNA. Ribosomal RNA contamination was evaluated by RNA pico chip using a BioAnalyzer (Agilent, Santa Clara, CA). The resulting mRNA was then used to prepare cDNA libraries using the RNA sequencing sample preparation kit (Illumina, San Diego, CA). Sample1 and Sample2 were sequenced by Illumina Genome Analyzer and then Genome Analyzer II, which generated four datasets: S1-R1, S2-R1, and S1-R2, S2-R2, respectively.

\section{Data filtering, mapping reads and identifying transcriptome contents}

The sequences generated went through a filtering process first. Any reads that contained numerous interspersed Ns in their sequences, or had relatively short reads $(<17 \mathrm{bp})$, were removed for the following analysis. Sequence reads obtained after quality control with filtering were analyzed using CLC Genomics Workbench 4 (CLC bio, Cambridge, MD). After mapping, the gene expression level was quantified by simply dividing the number of reads mapped to each gene by the size of its transcripts, commonly known as the number of reads per kilobase of exon per million mapped reads (RPKM) [2], for all 15,742 annotated chicken genes in the database. The gene expression level was then $\log _{2}$ transformed.

\section{Random sampling of S1-R2 and S2-R2}

We have obtained RNA-Seq data in three different levels of depth: $1.6 \mathrm{M}, 4.9 \mathrm{M}$, and about $30 \mathrm{M}$ reads. Clearly, there was a big gap between $4.9 \mathrm{M}$ and $30 \mathrm{M}$ reads. In order to identify the appropriate depth of transcriptome per sample that is sufficient for whole genome transcriptome profiling, it is important to generate additional datasets at different levels of depth. It would 
be very costly to re-sequence each sample to generate RNA-Seq data at different levels of sequencing depth. Random sampling from current dataset might provide a cost-effective approach for this purpose. This procedure synthetically created samples from the originally sequenced samples. Thus, for samples S1-R2 and S2-R2 data sets, by drawing without replacement a fixed number of reads from the overall data set, we randomly selected $10 \mathrm{M}, 15 \mathrm{M}$ and finally $20 \mathrm{M}$ reads. These random selections are repeated 4 times, resulting in total of 24 technical replicates with different transcriptome depth. Each one of the reads in the FASTQ format of the input files, which were used in the sampling, was selected equally likely. A program in Perl was written to serve this purpose. Then, the resulting replicate datasets were uploaded into CLC Genomics Workbench in the FASTQ format for the analysis individually. The correlation coefficients of gene expression (RPKMs) between replicates of each sequencing depth were calculated by JMP (SAS, Cary, NC). The average RPKMs of transcripts identified by each sequencing depth $(10 \mathrm{M}, 15 \mathrm{M}$ and 20 $\mathrm{M})$ were calculated to represent the gene expression values for further analysis.

\section{Correlation coefficients between different sequencing depths from the same sample}

In order to evaluate how reliable the sequence data is at each level of sequencing depth, correlation coefficients between lower and high depth sequence data for each sample were calculated by JMP (SAS, Cary, NC). Any genes with no gene expression at either sequence dataset were excluded from the correlation coefficient computation. The depth of sequencing is highly correlated with the abundance of gene expression, so we divided genes into the four quartile groups based on expression levels for each dataset, from the bottom $25 \%$ ( $1^{\text {st }}$ quartile) to the top $25 \%$ ( $4^{\text {th }}$ quartile). Subsequently, correlation coefficients between lower-depth sequence data and the data with 28.7- 29.6 M reads within each of the four quartile groups were calculated.

\section{Results}

\section{RNA-Seq for CDNA libraries}

The two chicken cDNA libraries (Sample1 and Sample2) were sequenced by the Illumina Genome Analyzer, which generated 4.9 M (60 bp) reads (S1-R1) and 1.6 M (60 bp) reads (S2-R1), respectively. Then, two technical replicate cDNA libraries from the same RNA samples were re-sequenced using the Genome Analyzer II, which generated 29.6 M (75 bp) reads (S1-R2) and 28.7 M (75 bp) reads (S2-R2).

\section{Random sampling of the S1-R2 and S2-R2}

The datasets of S1-R2 $(29.6 \mathrm{M})$ and S2-R2 $(28.7 \mathrm{M})$ were each randomly re-sampled into $10 \mathrm{M}, 15 \mathrm{M}$, and
$20 \mathrm{M}$ reads with four replicates each. The correlation coefficients between every two replicates for each resampled level (10 M, $15 \mathrm{M}$ and $20 \mathrm{M}$ ) within each sample (S1-R2 and S2-R2) were all greater than 0.98, which demonstrated that the sampling procedure is consistent and accurate. Averages of gene expression from the four replicates at each re-sampled level for each sample were used for further analysis.

\section{Effects of sequence depth on the distributions of transcripts}

The distributions of transcript abundance at different levels of sequence depth from Sample1 and Sample2 are presented in Figures 1 and 2, respectively. In general, the median and $75 \%$ percentile were similar across five different levels of depth, while the $95 \%$ percentile showed a slight increase, especially from $20 \mathrm{M}$ to $30 \mathrm{M}$. Significant decreases at the $25 \%$ and $5 \%$ percentile were observed, especially from $20 \mathrm{M}$ to $30 \mathrm{M}$. In addition, a significant decrease was also observed from $1.6 \mathrm{M}$ to 10 $\mathrm{M}$ in Sample2.

\section{Coverage of annotated chicken genes}

There are about 15,742 annotated chicken genes in the NCBI database. Number of detected chicken genes at different levels of sequencing depth from Samples 1 and 2 are presented in Table 1 . There were 14,336 genes detected in S1-R2 (29.6 M) and 14,212 genes in S2-R2 $(28.7 \mathrm{M})$, which accounted for $91.07 \%$ and $90.28 \%$ of all 15,742 annotated chicken genes in the database, respectively. With the reduction of sequencing depth, the number of detectable genes also decreased from $91 \%$ to $78 \%$ in Sample1 (Fig. 3A), and from 90\% to 68\% in Sample2 (Fig. 3B). Two significant drops of transcriptome coverage were observed: from $30 \mathrm{M}$ to $20 \mathrm{M}$, and $10 \mathrm{M}$ to $1.6 \mathrm{M}$.

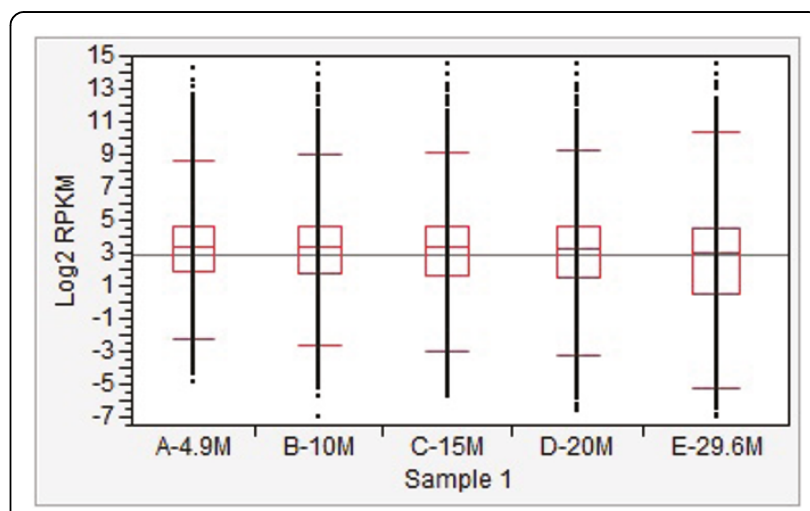

Figure 1 Distributions of $\log 2$ transformed reads of transcripts at different sequencing depths for Sample1. 


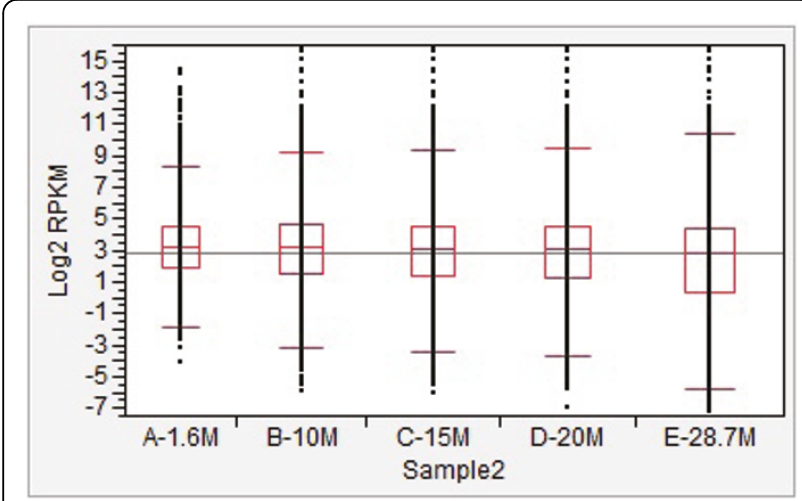

Figure 2 Distributions of $\log 2$ transformed reads of transcripts at different sequencing depths for Sample2.

\section{Correlation coefficients between different sequencing depths}

To evaluate the appropriate depth of sequence that is needed for transcriptome profiling, correlation coefficients between different levels of sequencing depth and the most abundant reads for each sample were calculated. For Sample1, overall correlation coefficients at four different levels of depth were greater than 0.95 . When we examined the four quartile groups based on expression level (Fig. 4A), correlation coefficients ranged from 0.34 to 0.67 for the $1^{\text {st }}$ quartile, 0.22 to 0.77 for the $2^{\text {nd }}$ quartile, 0.65 to 0.95 for the $3^{\text {rd }}$ quartile, and 0.97 to 1.0 for the $4^{\text {th }}$ quartile. A similar pattern in terms of correlation coefficient change was observed for the $1^{\text {st }}, 2^{\text {nd }}$, and $3^{\text {rd }}$ quartiles; a significantly increased correlation coefficient from $4.9 \mathrm{M}$ to $10 \mathrm{M}$, and kept relative flat from $10 \mathrm{M}$ to $20 \mathrm{M}$. For the $4^{\text {th }}$ quartile, correlation coefficients at four different levels of depth were greater than 0.95 . From the $1^{\text {st }}$ to the $4^{\text {th }}$ quartiles, there were significant increases for correlation coefficients between every two quartile groups $(p<0.01)$. For Sample2, overall correlation coefficients at four different levels of depth were greater than 0.98 , except for $1.6 \mathrm{M}$ at 0.84 . Correlation coefficients ranged from 0.08 to 0.72 for the $1^{\text {st }}$ quartile, 0.05 to 0.78 for the $2^{\text {nd }}$ quartile, 0.31 to 0.95 for the $3^{\text {rd }}$ quartile, and 0.87 to 1.0 for the $4^{\text {th }}$ quartile (Fig. 4B). The same pattern in terms of

Table 1 Numbers of detected annotated chicken genes at different levels of sequence depth.

\begin{tabular}{lclc}
\hline & Sample1 & \multicolumn{2}{c}{ Sample2 } \\
\hline & Numbers of genes & & Numbers of genes \\
\hline $\mathbf{2 9 . 6} \mathbf{M}$ & 14,336 & $\mathbf{2 8 . 7} \mathbf{~ M}$ & 14,212 \\
$\mathbf{2 0} \mathbf{~ M}$ & 13,011 & $\mathbf{2 0} \mathbf{~ M}$ & 12,895 \\
$\mathbf{1 5} \mathbf{M}$ & 12,822 & $\mathbf{1 5} \mathbf{~ M}$ & 12,690 \\
$\mathbf{1 0} \mathbf{M}$ & 12,515 & $\mathbf{1 0} \mathbf{~ M}$ & 12,406 \\
$\mathbf{4 . 9} \mathbf{M}$ & 12,276 & $\mathbf{1 . 6} \mathbf{~ M}$ & 10,664 \\
\hline
\end{tabular}

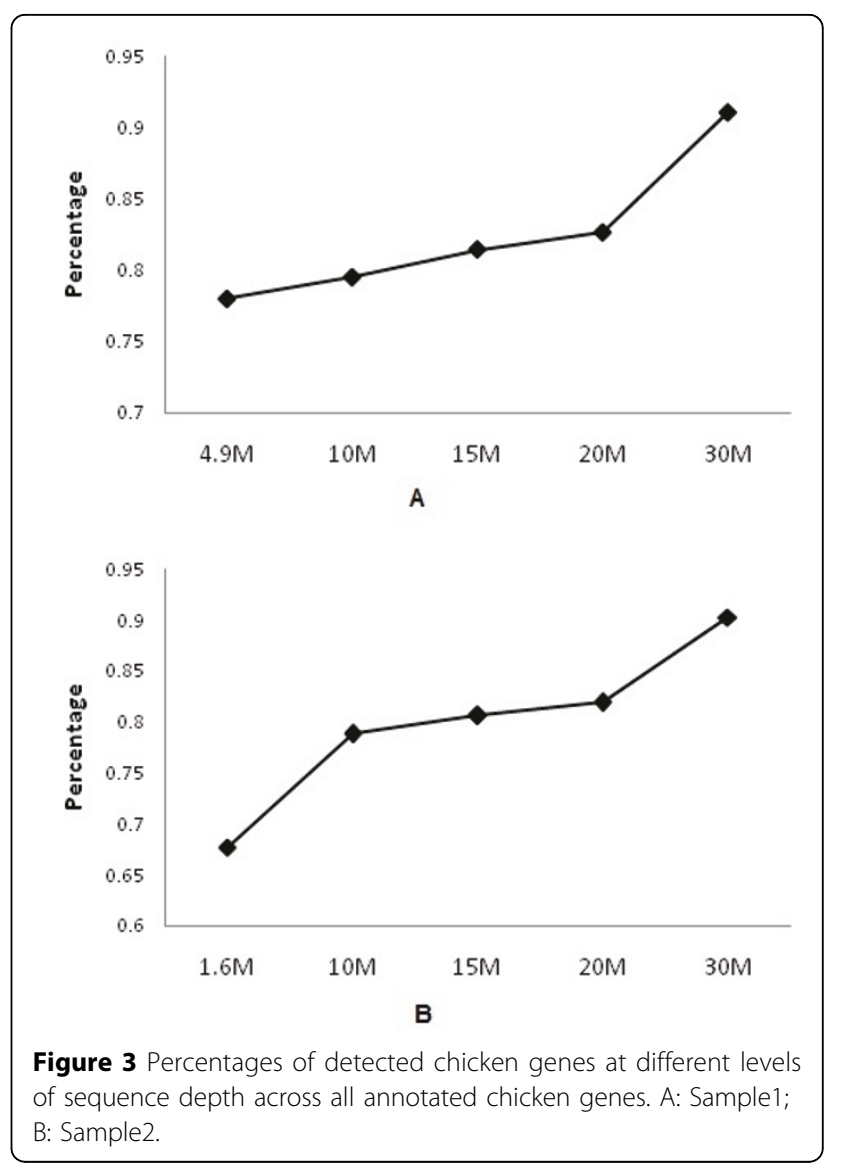

correlation coefficients changes at different levels of depth between Sample1 and Sample2 was also observed.

\section{Discussion}

In the current study, RNA-Seq was performed twice using two chicken cDNA samples. The first run of RNA-Seq had fewer number of reads and larger variation in terms of total number of reads between the two samples, while the second run had greater number of reads and very small variation between the two samples. The first run was performed at the very early stage of the sequencing technology when it was still in the testing phase. The lower reads and larger variation in the first run may be coming from two major sources of technical error: the purification of cDNA templates during the library preparation, and the loading of libraries onto flow cells (RNA-Seq technical guide and personal communications, Illumina technical support staff). These potential sources of errors were corrected during the second RNA-Seq analysis, which provided very good sequencing depth with greater number of reads. The first RNA-Seq datasets were directly derived from actual experiment, which made the results more informative than replicating datasets by random sampling. 


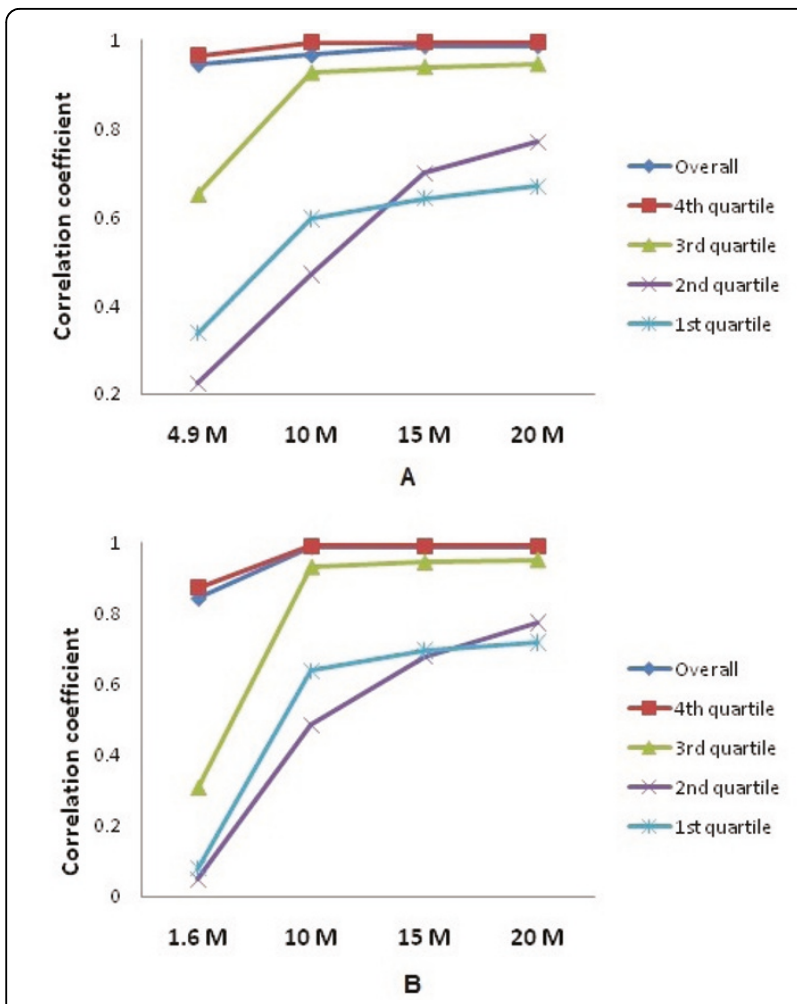

Figure 4 Correlation coefficients between $30 \mathrm{M}$ reads and four different levels of sequence depth at different quartiles. A: Sample1; B: Sample2.

Therefore, we chose to include these two early datasets in the analysis in the current study. Furthermore, all of the reads from each sample were normalized by the RPKM and the datasets can serve as a reference for random sampling at different sequencing depths from the exact same samples.

The capacity of sequencing length of $60 \mathrm{bp}$ for the first run was increased to $75 \mathrm{bp}$ at the second RNA-Seq analysis. Longer reads should reduce estimation error and mapping uncertainty, and read lengths have been consistently increasing with improving Illumina massively parallel sequencing technology. However, people have noted that the number of reads is more important than the read length once reaching a minimum read length of $25 \mathrm{bp}[9,10]$. The read lengths $(60 \mathrm{bp}$ and 75 bp) in the current study were larger than $25 \mathrm{bp}$; therefore, the read length will not affect the overall conclusions drawn.

As a powerful new technology for transcriptome analysis, RNA-Seq provides a more comprehensive view of the transcriptome than earlier technologies. Besides its ability to detect splicing variation, RNA editing and discovery of new transcripts [11], RNA-Seq can also function in the role of a conventional microarray in measuring gene expression due to its accurate measurements. In order to detect less abundant transcripts, appropriate sequencing depth is needed. The transcriptome coverage or sequencing depth needed for a given study can be affected by several factors such as genome size, transcriptome complexity and objectives of the study. In general, the more complex the transcriptome, the more sequencing depth is required for adequate coverage [12]. Depending on the purpose of transcriptome analysis, the requirement of sequencing depth varies. In most transcriptome studies, quantifying mRNA abundance is one of the major objectives. There is a certain sequencing depth that is sufficient in simple transcriptomes. For example, in the yeast genome, a 29.9 M (35 bp) reads dataset was generated by RNA-Seq which was able to get $100 \%$ transcriptome coverage [13]. The number of transcripts detected by RNA-Seq in the yeast dataset was able to reach $80 \%$ transcriptome coverage at $4 \mathrm{M}$ mapped reads, and even though the sequencing depth doubled as $8 \mathrm{M}$ reads, the transcriptome coverage only increased $10 \%[13,14]$. These results suggest that the improvement of sequencing depth or transcriptome coverage after reaching a certain sequencing depth had relatively less impact on detecting low abundant genes [15]. In addition, the cost per sample per lane by RNA-Seq is still not affordable for most laboratories. Recent development in multiplex labelling using bar-coded libraries by Illumina and continued increase in sequence output have made it possible to sequence multiple samples per lane without extra cost or running time [16]. Therefore, it is imperative to examine the correlation between sequencing depth and transcriptome coverage; in other words, what sequencing depth might be sufficient in reaching a certain level of transcriptome coverage and reliable measurement for RNA-Seq. In order to accomplish this objective, two approaches could be applied: experimental or simulation methods. Both methods have been applied in this study. High correlation among replicates within each sequencing depth, gradual increase in correlation coefficients from $10 \mathrm{M}$ to $20 \mathrm{M}$, and consistent patterns observed between Samples1 and 2 (Fig. 4) have demonstrated that random sampling was an effective and reliable method in reaching the goals of this study.

Transcriptome coverage is one of the most important parameters in profiling global gene expression. The number and level of transcript isoforms is not always known and transcription activity varies across the genome [14]. This was confirmed in a study by using the number of unique transcription start sites as a measure of coverage in mouse cells [15]. In the current study, we took a more practical approach using all annotated genes in the chicken genome. Because the chicken genome is far under-annotated, we assume that the 15,742 annotated chicken genes in the database would well 
represent different levels of expression abundance in the chicken genome, which is essential for the analysis of transcriptome coverage in this study. Since gene expression depends on tissue and time of biological process [15], it is impossible for any single tissue to have all genes in the genome expressed. Ninety percent of all annotated genes (Fig. 3) detected at about $30 \mathrm{M}$ (75 bp) reads might represent a saturated detection of the whole genome. The analysis results indicated significant improvements of transcriptome coverage occurred from 1.6 $\mathrm{M}$ to $4.9 \mathrm{M}$ and from $20 \mathrm{M}$ to $30 \mathrm{M}$. Depending on the purpose of transcriptome analysis, the current study suggested that $10 \mathrm{M}(75 \mathrm{bp})$ reads could have $80 \%$ of transcriptome coverage, while $30 \mathrm{M}(75 \mathrm{bp})$ reads could reach $90 \%$ of transcriptome coverage.

When we analyzed overall correlation coefficients at different levels of sequencing depth regardless of gene expression level, we observed very high correlation coefficients between each level of sequencing depth compared with $30 \mathrm{M}$, except for $1.6 \mathrm{M}$. One might draw a conclusion that there is no significant difference among different levels of sequencing depth. But as we see in Figure 4, this might be true in the case of highly abundant genes (the $4^{\text {th }}$ quartile group), but not in the case of the $1^{\text {st }}$ to $3^{\text {rd }}$ quartile groups, especially the first two quartile groups (i.e. expression below the median). High abundant genes will be less affected by sequencing depth than low abundant genes, because high abundant genes are more likely to be captured even when the sequencing depth varies [17]. This is also confirmed by our analysis. Collectively, the following points can be inferred: 1) Sequencing depth below $20 \mathrm{M}$ (75 bp) reads had a significant effect on detecting transcript levels of medium and low abundant transcripts; 2) Sequencing depth at both $1.6 \mathrm{M}$ and $4.9 \mathrm{M}$ would result in unreliable mRNA expression on all genes except highly abundant transcripts; 3) There was no significant improvement in correlation coefficients when sequencing depth doubled from $10 \mathrm{M}$ to $20 \mathrm{M}$. Based on these analysis, the results suggested: 1) $5 \mathrm{M}$ reads might be sufficient in obtaining reliable mRNA expression measurement on highly abundant transcripts; 2) When sequencing depth is beyond $10 \mathrm{M}$ reads, a relatively reliable measurement of mRNA expression is expected, especially for abundant transcripts; 3 ) It seems that 30 $M$ of reads is needed to achieve reliable measurement of mRNA expression across all genes in the chicken genome. To our knowledge, this is the first study evaluating the appropriate sequencing depth using RNA-Seq in farm animals and will provide the first reference for similar studies. The knowledge generated from this study has laid a solid foundation for applying this analysis to other species.

\section{Conclusions}

In summary, the analysis from this study demonstrated that $30 \mathrm{M}(75 \mathrm{bp})$ reads is sufficient to detect all annotated genes and provide a reliable measurement of mRNA abundance in chicken lungs using RNA-Seq. As we expected, the depth of sequencing had significant impact on low abundance transcripts, but not on high abundance transcripts. In practice, if RNA-Seq serves as a replacement of microarray technology, $10 \mathrm{M}$ (75 bp) reads would allow detection of about $80 \%$ of annotated chicken genes. Finally, increasing the depth of sequencing from $10 \mathrm{M}$ to 20 $M$ reads did not have a significant effect on transcriptome coverage and reliability of mRNA measurements, whereas $30 \mathrm{M}$ reads was needed to achieve reliable measurement of mRNA expression across all genes in the genome.

\section{Acknowledgements}

We are grateful for Dr. Jason W. Abernathy for his helps for revising the manuscript. This project was supported by the Formula Animal Health Grant no. 07-024 from the USDA Cooperative State Research, Education, and Extension Service.

This article has been published as part of BMC Bioinformatics Volume 12 Supplement 10, 2011: Proceedings of the Eighth Annual MCBIOS

Conference. Computational Biology and Bioinformatics for a New Decade. The full contents of the supplement are available online at http://www. biomedcentral.com/1471-2105/12?issue=S10.

\section{Author details}

'Department of Poultry Science, Texas A \&M University College Station, TX 77843-2472, USA. ${ }^{2}$ Agrilife Genomics and Bioinformatics Services, Texas A\&M University, College Station, TX 77843-2312, USA. ${ }^{3}$ Department of Electrical and Computer Engineering, Texas A\&M University, College Station, TX 77843, USA. ${ }^{4}$ Department of Molecular and Human Genetics, Baylor College of Medicine, Houston, TX 77030, USA.

\section{Authors' contributions}

YW analyzed the data and drafted the manuscript, NG wrote the code for sampling the RNA-Seq data, CJ and UB contributed ideas for the data analysis and revised the manuscript, HW and RC ran RNA-Seq samples, and $\mathrm{HZ}$ designed the experiment, provided the concept of the analysis. All authors submitted comments, read and approved the final manuscript.

\section{Competing interests}

The authors declare that there are no competing interests.

Published: 18 October 2011

\section{References}

1. Blencowe BJ, Ahmad S, Lee L: Current-generation high-throughput sequencing: deepening insights into mammalian transcriptomes. Genes Dev 2009, 23(12):1379-1386.

2. Mortazavi A, Williams BA, McCue K, Schaeffer L, Wold B: Mapping and quantifying mammalian transcriptomes by RNA-Seq. Nat Methods 2008, 5(7):621-628.

3. Li X, Chiang HI, Zhu J, Dowd SE, Zhou H: Characterization of a newly developed chicken 44K Agilent microarray. BMC Genomics 2008, 9:60.

4. Cassone M, Giordano A, Pozzi G: Bacterial DNA microarrays for clinical microbiology: the early logarithmic phase. Front Biosci 2007, 12:2658-2669.

5. Okoniewski MJ, Miller CJ: Hybridization interactions between probesets in short oligo microarrays lead to spurious correlations. BMC Bioinformatics 2006, 7:276. 
6. Draghici S, Khatri P, Eklund AC, Szallasi Z: Reliability and reproducibility issues in DNA microarray measurements. Trends Genet 2006 22(2):101-109.

7. Wang L, Feng Z, Wang $X$, Zhang X: DEGseq: an R package for identifying differentially expressed genes from RNA-seq data. Bioinformatics 2010 26(1):136-138.

8. Liu S, Lin L, Jiang P, Wang D, Xing Y: A comparison of RNA-Seq and highdensity exon array for detecting differential gene expression between closely related species. Nucleic Acids Res 2011, 39(2):578-588.

9. Li B, Ruotti V, Stewart RM, Thomson JA, Dewey CN: RNA-Seq gene expression estimation with read mapping uncertainty. Bioinformatics 2010, 26(4):493-500.

10. Nicolae M, Mangul S, Mandoiu II, Zelikovsky A: Estimation of alternative splicing isoform frequencies from RNA-Seq data. Algorithms Mol Biol 2011, 6(1):9.

11. Wilhelm BT, Briau M, Austin P, Faubert A, Boucher G, Chagnon P, Hope K, Girard S, Mayotte N, Landry JR, et al: RNA-seq analysis of 2 closely related leukemia clones that differ in their self-renewal capacity. Blood 2011, 117(2):e27-38.

12. Cloonan N, Forrest AR, Kolle G, Gardiner BB, Faulkner GJ, Brown MK, Taylor DF, Steptoe AL, Wani S, Bethel G, et al: Stem cell transcriptome profiling via massive-scale mRNA sequencing. Nat Methods 2008, 5(7):613-619.

13. Nagalakshmi U, Wang Z, Waern K, Shou C, Raha D, Gerstein M, Snyder M: The transcriptional landscape of the yeast genome defined by RNA sequencing. Science 2008, 320(5881):1344-1349.

14. Wang Z, Gerstein M, Snyder M: RNA-Seq: a revolutionary tool for transcriptomics. Nat Rev Genet 2009, 10(1):57-63.

15. Wilhelm BT, Marguerat S, Watt S, Schubert F, Wood V, Goodhead I, Penkett CJ, Rogers J, Bahler J: Dynamic repertoire of a eukaryotic transcriptome surveyed at single-nucleotide resolution. Nature 2008 453(7199):1239-1243.

16. Stiller M, Knapp M, Stenzel U, Hofreiter M, Meyer M: Direct multiplex sequencing (DMPS)-a novel method for targeted high-throughput sequencing of ancient and highly degraded DNA. Genome Res 2009, 19(10):1843-1848.

17. Ramskold D, Wang ET, Burge CB, Sandberg R: An abundance of ubiquitously expressed genes revealed by tissue transcriptome sequence data. PLoS Comput Biol 2009, 5(12):e1000598.

doi:10.1186/1471-2105-12-S10-S5

Cite this article as: Wang et al:: Evaluation of the coverage and depth of transcriptome by RNA-Seq in chickens. BMC Bioinformatics 201112 (Suppl 10):S5.

\section{Submit your next manuscript to BioMed Central and take full advantage of:}

- Convenient online submission

- Thorough peer review

- No space constraints or color figure charges

- Immediate publication on acceptance

- Inclusion in PubMed, CAS, Scopus and Google Scholar

- Research which is freely available for redistribution 